\title{
Contribution d'un modèle réduit physique aux études d'ingénierie de l'ascenseur à poissons de Golfech
}

\author{
A scale model contributing to the engineering studies \\ of the Golfech fish lift
}

\author{
Ch. Puyo \\ A. Venel
}

Sogreah

6, rue de Lorraine, 38130 Echirolles

Un modèle réduit à l'échelle $1 / 10$ du bassin d'attrait à l'ascenseur à poissons du barrage hydroélectrique de Golfech a été réalisé en 1984 au Laboratoire de Sogreah pour le compte de la Direction de l'Equipement d'EDF/REAM.

Malgré les contraintes sévères imposées à l'hydraulique par le génie civil, un ensemble de dispositifs satisfaisants a pu être mis au point qui dissipe convenablement l'énergie de la chute, répartit normalement les débits dans les bassins d'alimentation, alimente les canaux d'amenée aux sorties latérales et le bassin de stabulation, avec des vitesses d'écoulement compatibles avec le cahier des charges.

Le dispositif proposé est performant sans manipulations d'organes de réglage, autres que les vannes au droit des sorties.

A 1/10 scale reduced model of the attraction basin with a fish lift at Golfech hydraulic dam was built in 1984 at the Sogreah laboratory for the Equipment Management of EDF.

Despite the severe constraints imposed upon hydraulics by civil engineering, a complete set of suitable devices have been built and which approximately dissipate the energy of the waterfall, normally distribute the flow rates in the feeding basins, supply the feeding channels at the lateral outlets and the housing basins, with flow rates complying to the specifications sheet.

The proposed device is high-performance and does not require any handling of adjustment devices apart from the valves at the right of the outlets.

\section{Introduction}

Lors de l'élaboration de l'avant-projet sommaire de l'ascenseur à poissons de Golfech, il est rapidement apparu que deux points particuliers conditionnaient le succès ou l'échec du fonctionnement de l'ouvrage :

- le jet constitué par le débit d'attrait au débouché dans le canal de fuite,

- la distribution des débits à l'intérieur du bassin d'attrait.
Les caractéristiques du jet d'attrait ont été définies sur modèle réduit au laboratoire de Banlève de l'Institut de mécanique des fluides de Toulouse.

Les formes et dispositifs du bassin d'attrait ont été étudiés sur modèle réduit au Laboratoire de Sogreah à Grenoble.

L'objectif était triple :

- respecter les vitesses fixées a priori, dans le jet d'attrait et dans les différents compartiments du bassin,

- obtenir des résultats acceptables avec le minimum de manipulations d'organes de réglage, 


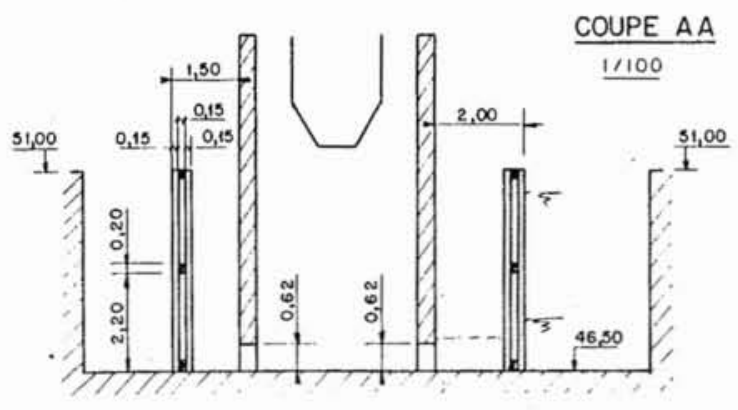

BASSIN D'ATTRAIT A L'ASCENSEUR
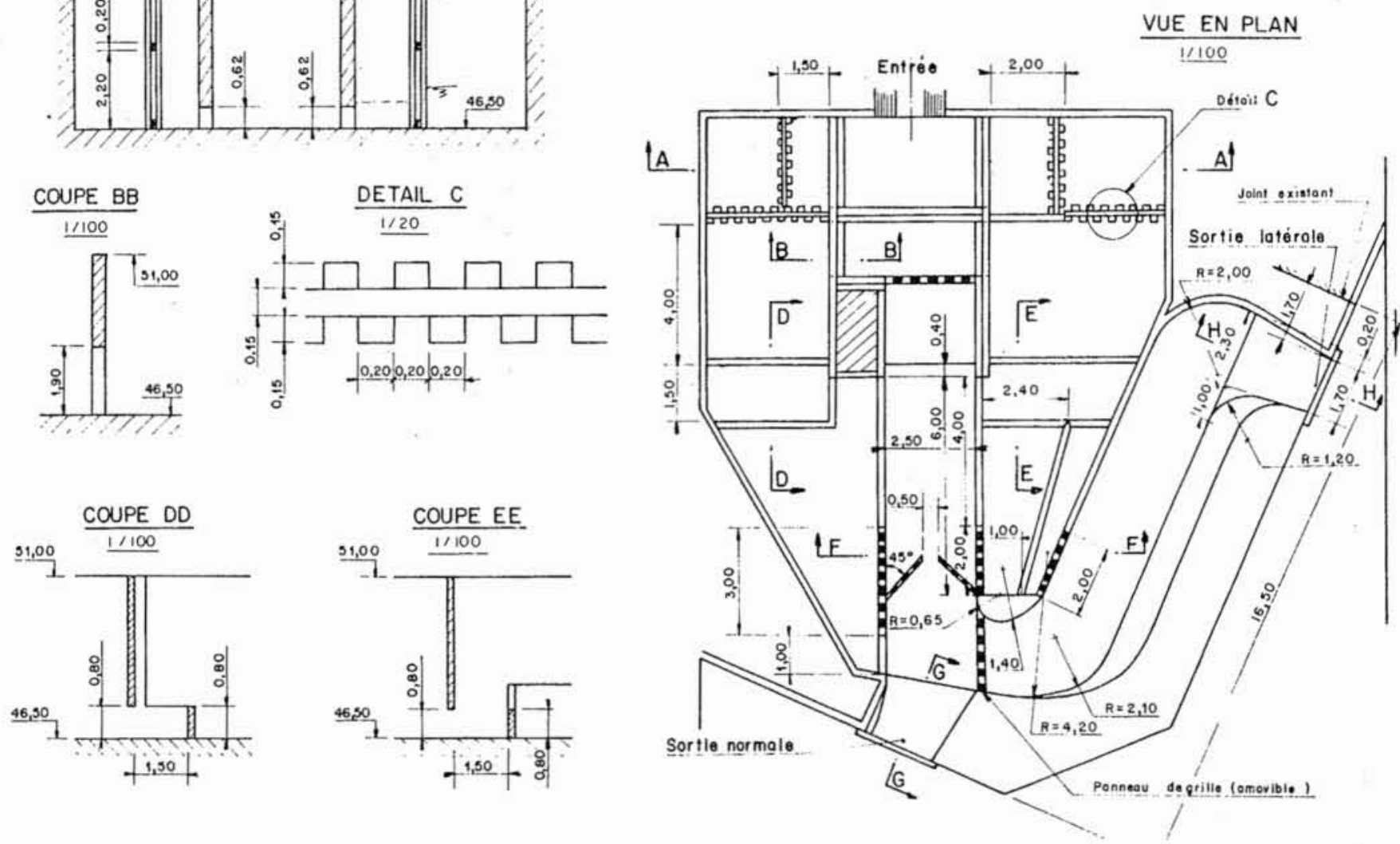

Figure 1

- simplifier les formes de l'ouvrage pour en faciliter la construction.

Seul, un modèle réduit physique à une échelle de l'ordre du $1 / 10$ pouvait permettre de satisfaire avec certitude ces trois objectifs.

\section{Le modèle réduit physique}

Le bassin d'attrait a été réalisé à l'échelle du $1 / 10$, en contre-plaqué marine, entre deux bassins construits " en dur " :

- le bassin d'alimentation où les débits étaient mesurés (3 à $10 \mathrm{~m}^{3} / \mathrm{s}$ nature, soit 10 à $32 \mathrm{l} / \mathrm{s}$ modèle),

- le bassin aval représentant les niveaux en Garonne variant de 47.60 à $50.00 \mathrm{NGF}$.
Le choix du contre-plaqué a permis de réaliser très rapidement, en cours d'essais, les multiples modifications apportées au modèle lors de la mise au point des formes.

Les mesures ont concerné :

- les débits à l'amont du bassin d'attrait,

- les niveaux dans le bassin d'attrait et dans le bassin aval,

- les vitesses moyennes dans les divers compartiments du bassin.

Les dimensions de l'ouvrage sont données sur la figure 1 ci-dessus.

\section{Fonctionnement hydraulique du bassin d'attrait}

Le schéma de la figure 2 permet de comprendre le fonctionnement du bassin d'attrait brièvement rappelé ci-après (de l'aval vers l'amont, dans le sens du déplace- 
Tableau 1. - Conditions d'essai du bassin d'attrait de l'ascenseur à poissons.

\begin{tabular}{|c|c|c|c|c|c|c|c|c|c|}
\hline $\mathrm{N}^{\circ}$ de & 1'essa1 & $\begin{array}{c}\text { Débit } \\
\text { d'alimen- } \\
\text { tation } \mathrm{m}^{3} / \mathrm{s}\end{array}$ & $\begin{array}{c}\text { Débit } \\
\text { sort } 1 \mathrm{c} \\
\text { normale } \mathrm{m}^{3} / \mathrm{s}\end{array}$ & $\begin{array}{l}\text { Déblt } \\
\text { sortie } 1 \text { a- } \\
\text { térale } \mathrm{m}^{3} / \mathrm{s}\end{array}$ & $\begin{array}{c}\text { Niveau } \\
\text { aval bassin } \\
\text { NCF }\end{array}$ & $\begin{array}{l}\text { Niveau dans } \\
\text { bassin de } \\
\text { st abulation }\end{array}$ & $\begin{array}{c}\text { Débit du } \\
\text { barrage } \\
\mathrm{m}^{3} / \mathrm{s}\end{array}$ & $\begin{array}{l}\text { Cote vanne } \\
\text { sortie } \\
\text { normale Nef }\end{array}$ & $\begin{array}{l}\text { Cute vaune } \\
\text { surtle } \\
\text { latérale Nof }\end{array}$ \\
\hline & 1 & 5 & 5 & 0 & 50,00 & 50,33 & 1000 & 48.55 & fcrmée \\
\hline & 2 & 5 & 5 & 0 & 49,30 & 49.62 & 600 & 47,85 & fermée \\
\hline & 3 & 5 & s & 0 & 48,50 & 48,80 & 300 & 47,00 & fe rtaíe \\
\hline & 4 & 5 & 5 & 0 & 47,60 & 47,90 & 100 & "effacée" & ferméc \\
\hline & 5 & 5 & 0 & 5 & 47,60 & 48,14 & 100 & fermée & "effacce" \\
\hline & 6 & 5 & 0 & 5 & 48,50 & 48,33 & 300 & fermúe & 46.90 \\
\hline & 7 & 5 & 0 & 5 & 49.30 & 49,61 & 600 & fermée & 47,75 \\
\hline & 8 & 5 & 0 & 5 & 50,00 & 50,31 & 1000 & fermete & 48,45 \\
\hline & 9 & 3 & 0 & 3 & 47,60 & 47,90 & 100 & fermée & "effacéc" \\
\hline & 91 & 3 & 0 & 3 & 47,60 & 47,90 & 100 & fermée & "effacće" \\
\hline & $10^{2}$ & 3 & 0 & 3 & 48,50 & 48,83 & 300 & fermeic & 47,50 \\
\hline & 11 & 3 & 0 & 3 & 49,30 & 49,62 & 600 & fermće & 48,35 \\
\hline & 12 & 3 & 3 & 0 & 49,30 & 49,63 & 600 & 48,35 & fermite \\
\hline & 13 & 3 & 3 & 0 & 48,50 & 48,85 & 300 & 47,60 & formẻe \\
\hline & 133 & 3 & 3 & 0 & 48,50 & 48,85 & 300 & 47,60 & fernice \\
\hline & $14^{2}$ & 3 & 3 & 0 & 47,60 & 47,95 & 100 & 46,70 & feruce \\
\hline & 15 & 10 & 5 & 5 & 50,00 & 50,31 & 1000 & 48,50 & 48,40 \\
\hline & 16 & 10 & 5 & 5 & 49,30 & 49,65 & 600 & 47,80 & 47,70 \\
\hline & 17 & 10 & 5 & 5 & 48,50 & 48,85 & 300 & 46,90 & 46,80 \\
\hline
\end{tabular}

Tableau 2. - Vitesses dans le bassin d'attrait (en $\mathrm{m} / \mathrm{s}$ ). .

\begin{tabular}{|c|c|c|c|c|c|c|c|c|c|}
\hline \multirow[b]{3}{*}{$\mathbb{N}^{\circ} \mathrm{de}$} & \multirow[b]{3}{*}{ I'essal } & \multirow{3}{*}{$\begin{array}{l}\text { Sortie } \\
\text { normale }\end{array}$} & \multirow{3}{*}{$\begin{array}{l}\text { Sortie } \\
\text { latérale }\end{array}$} & \multirow{3}{*}{$\begin{array}{c}\text { Bassin } \\
\text { srabulation }\end{array}$} & \multirow[b]{3}{*}{ Nasse } & \multicolumn{4}{|c|}{ Canaux d'anenées } \\
\hline & & & & & & \multirow[b]{2}{*}{$\begin{array}{l}\text { Sortele } \\
\text { Normale }\end{array}$} & \multicolumn{3}{|c|}{ Sortie latérale } \\
\hline & & & & & & & Coude & anont & $\begin{array}{l}\text { Section } \\
\text { courante }\end{array}$ \\
\hline & 1 & 2,03 & 0 & 0,10 & 0,38 & 0.59 & & - & - \\
\hline & 2 & 2,09 & 0 & 0,17 & 0.52 & 0,82 & & - & - \\
\hline & 3 & 2,16 & 0 & 0,20 & 0,56 & 1,09 & & - & - \\
\hline & 4 & 2.37 & 0 & 0,42 & 1.20 & 2,10 & & - & - \\
\hline & 5 & 0 & 2,37 & 0,29 & 0,70 & - & & 1.57 & 2,11 \\
\hline & 6 & D & 2,13 & 0,12 & 0,45 & - & & 0,77 & 1,30 \\
\hline & 7 & 0 & 2,08 & 0,22 & 0,48 & - & & 0.72 & 0,75 \\
\hline & 8 & 0 & 2,08 & 0,10 & 0,40 & - & & 0,60 & 0,67 \\
\hline & 9 & 0 & 1,94 & 0,17 & 0,75 & - & & 1,40 & 1,37 \\
\hline & 93 & 0 & 1,94 & 0,27 & 0,77 & - & & 1.49 & 1.37 \\
\hline & $10^{2}$ & 0 & 2,07 & 0,11 & 0.29 & - & & 0.54 & 0.83 \\
\hline & 11 & 0 & 2,19 & 0.10 & 0.28 & - & & 0.45 & 0,45 \\
\hline & 12 & 1,96 & 0 & 0,13 & 0.35 & 0.52 & & - & - \\
\hline & 13 & 2,01 & 0 & 0,10 & 0,34 & 0,68 & & - & - \\
\hline & $13 \frac{1}{2}$ & 2,01 & 0 & 0,18 & 0,45 & 0,68 & & - & - \\
\hline & $14^{2}$ & 2,23 & 0 & 0,29 & 0,75 & 1,19 & & - & - \\
\hline & 15 & 2,16 & 1,95 & 0,16 & 0,81 & 0,82 & & 0,71 & 0,64 \\
\hline & 16 & 2,19 & 1,94 & 0.20 & 1,12 & 1.27 & & 0.39 & 1,02 \\
\hline & 17 & 2,26 & 1,90 & 0,23 & 1,17 & 1,48 & & 0,25 & 1.34 \\
\hline
\end{tabular}

ment du poisson migrateur) :

- un jet d'appel frontal et/ou latéral, réglable par des vannes télescopiques en fonction du niveau an Garonne (vitesse de l'ordre de $2,5 \mathrm{~m} / \mathrm{s}$ ),

- un canal d'amenée où la vitesse est comprise entre 0,5 et $2 \mathrm{~m} / \mathrm{s}$ selon les configurations,

- la nasse où la vitesse est comprise entre 0,3 et $1,2 \mathrm{~m} / \mathrm{s}$, - le bassin de stabulation où la vitesse est inférieure à 0,30 $\mathrm{m} / \mathrm{s}$.

Le débit, réglable, arrive par le canal d'entrée dans le puits de répartition des débits, puis dans deux bassins de dissipation latéraux.

Une partie du débit est dirigée vers le bassin de stabulation, et le rete se répartit entre les deux compartiments latéraux pour retourner au canal d'amenée à travers des grilles à l'aval de la nasse.

Le dispositif peut fonctionner (en réglant le débit amont) avec une seule ou les deux sorties en fonctionnement.

\section{Les essais}

Une vingtaine d'essais ont été réalisés dans les trois configurations suivantes :

- sortie normale seule,

- sortie latérale seule,

- sortie normale et latérale.

On a fait varier le débit d'alimentation, et donc le débit de chaque sortie, le niveau aval du bassin et les cotes de réglage des vannes équipant les sorties.

Les ouvertures des diverses passes ainsi que les formes ont été ajustées en cours d'essais, jusqu'à obtenir des vitesses satisfaisantes en chaque point important de l'ouvrage.

Les tableaux 1 et 2 ci-dessus récapitulent les conditions d'essais et les vitesses obtenues pour chacun des essais.

Pour illustrer les répartitions des débits et les variations de vitesses dans le bassin d'attrait, nous avons reporté sur des schémas les principaux résultats obtenus. Quelques- 


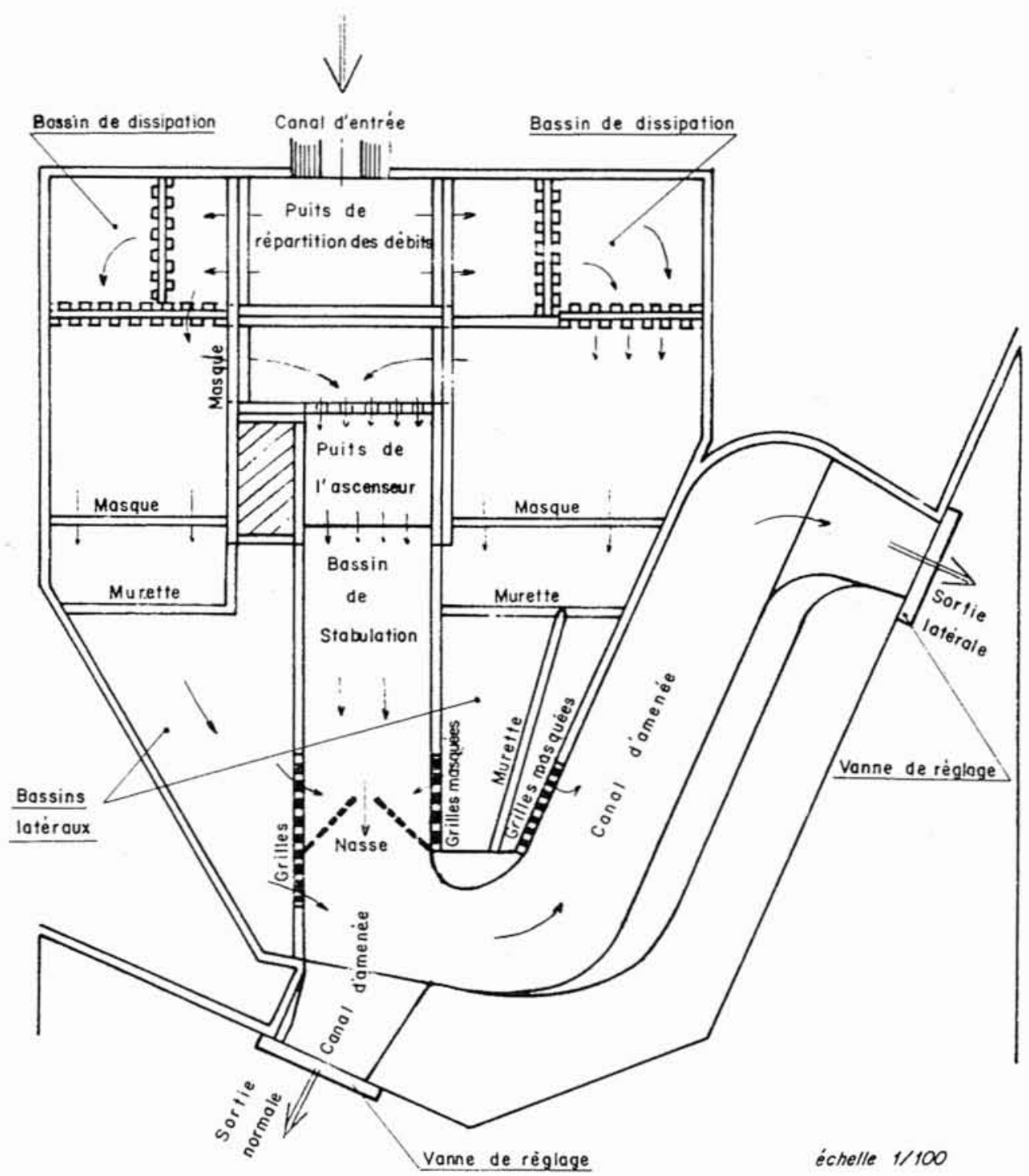

Figure 2. - Schéma descriptif du bassin d'attrait.

unes des configurations étudiées sont jointes en annexe (figures $n^{\circ} 3$ à 7 ).

\section{Résultats obtenus - Adaptations ultérieures de l'ouvrage}

Les essais sur modèle réduit physique ont montré qu'on pouvait obtenir, dans chacune des configurations étudiées, les vitesses souhaitables dans chaque compartiment du bassin d'attrait, sans prévoir de dispositifs de vannage autres que les vannes équipant les sorties.

Ce résultat est intéressant car il permet de diminuer le coût de l'ouvrage et de simplifier les manœuvres lors de la remontée des poissons. les essais en vraie grandeur effectués en 1985 ont montré que l'ouvrage fonctionnait correctement, sauf en ce qui concerne la progression des aloses dans le bassin de stabulation.

Il semble que la vitesse de $0,30 \mathrm{~m} / \mathrm{s}$ fixée a priori soit trop faible et que les aloses s'arrêtent trop près de l'entrée, avant d'avoir franchi la nasse.

Une augmentation de la vitesse dans le bassin de stabulation est possible en modifiant la répartition des débits à l'amont par obturation partielle des passes rive droite et rive gauche, comme illustré par la figure 5 par exemple.

A signaler également que les essais sur modèles réduits ont permis de définir les lois d'ouverture des vannes équipant les sorties en fonction du niveau aval dans le canal de fuite et pour la gamme de débit considérée. 
ESSAI N:1

Deut dentree $=5 \mathrm{mt} / \mathrm{s}$

Denat sortie normole $=5 \mathrm{~m} / \mathrm{s}$

Detat sortie laterole : $0 \mathrm{~m}^{2} \mathrm{~s}$

Cébit GARONALE $=1000 \mathrm{~m} / \mathrm{s}$

Nitrear gval $=50,00$

Entree

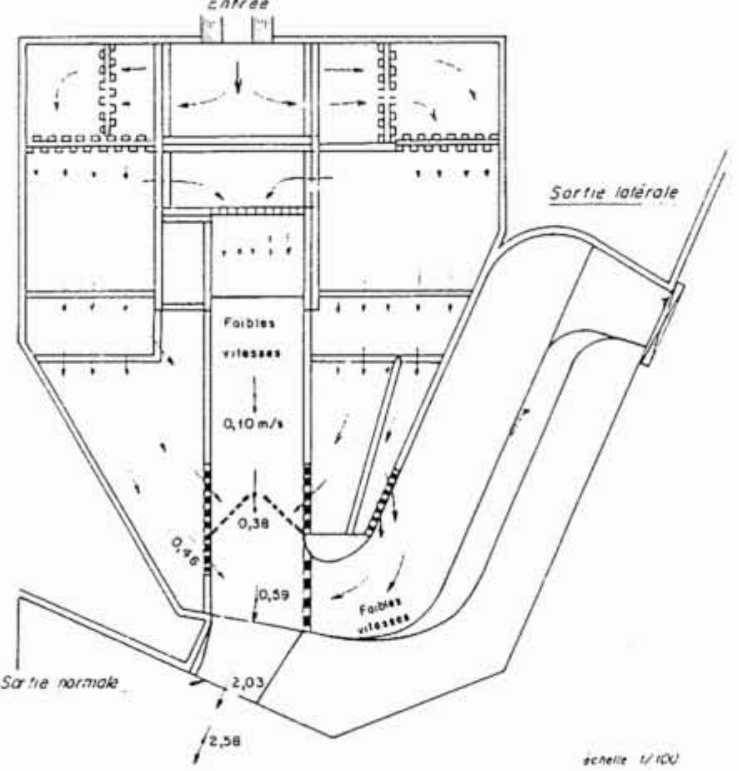

ESSAI N:2

Debil dentree $=2$ mits

Detit sortie normale $=5 \mathrm{~m}^{3}$

Debt sortie lateraie $=0 \mathrm{mis}$

CeBI GARUANE $=600 \mathrm{~m}^{2} \mathrm{~s}$

Therai jvat= 49,30

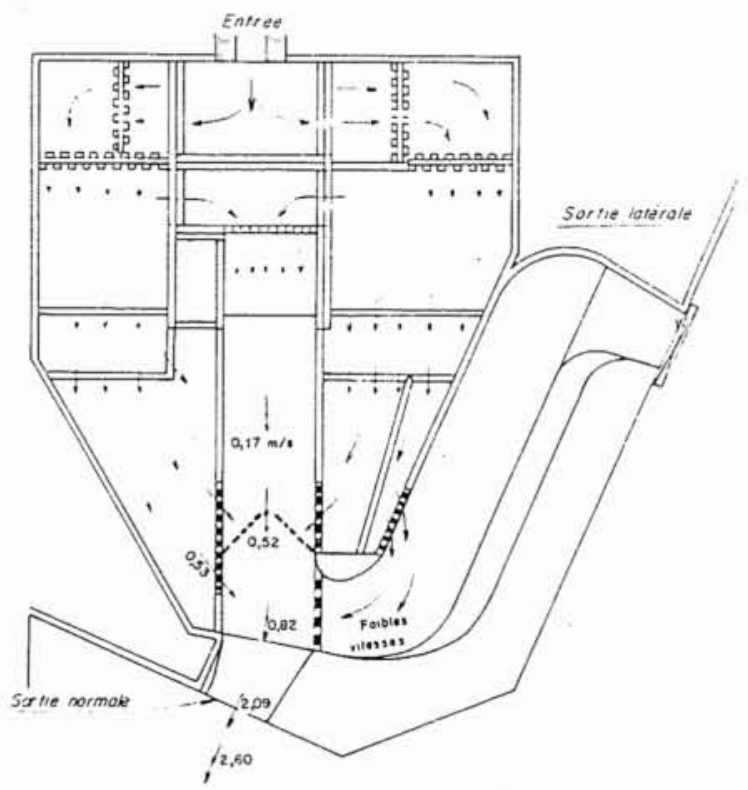

Figure 3

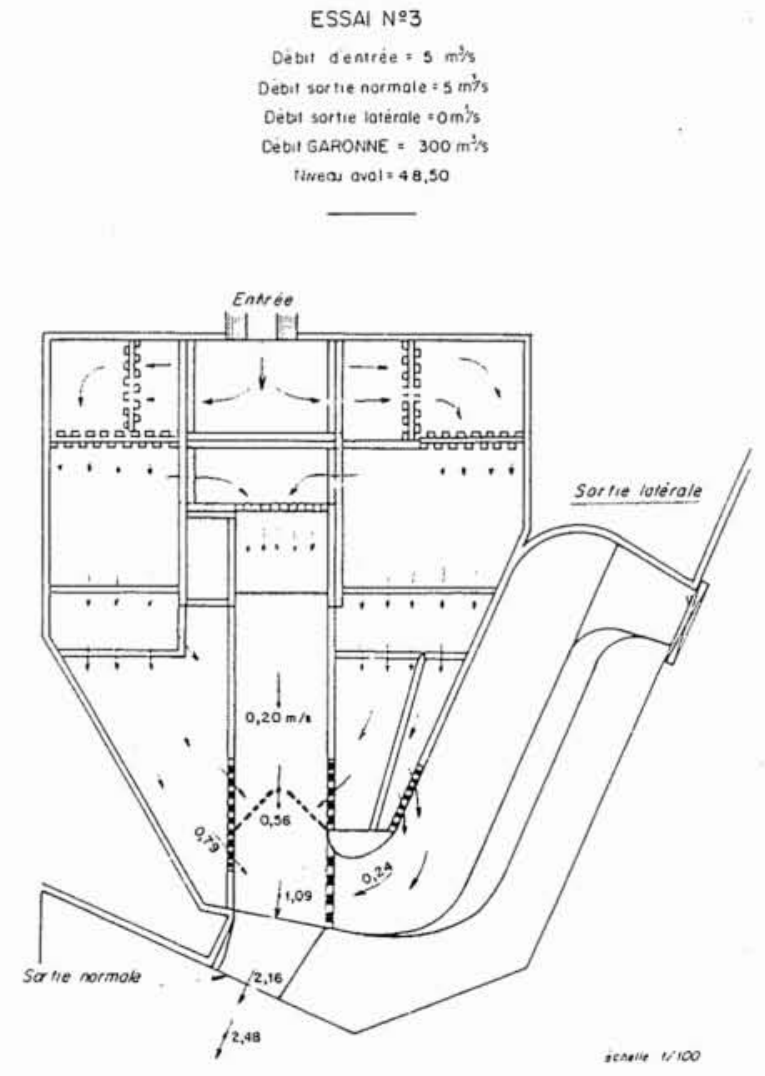

ESSAI N 24

Début dentree $=5 \mathrm{~m} / \mathrm{s}$

Debut sortie normale $=5 \mathrm{mis}$

Debat sortie laterole $=0 \mathrm{~m}^{2} / \mathrm{s}$

DEDII BARONNE $=100 \mathrm{~m} / \mathrm{s}$

Atrea aval $=47,60$

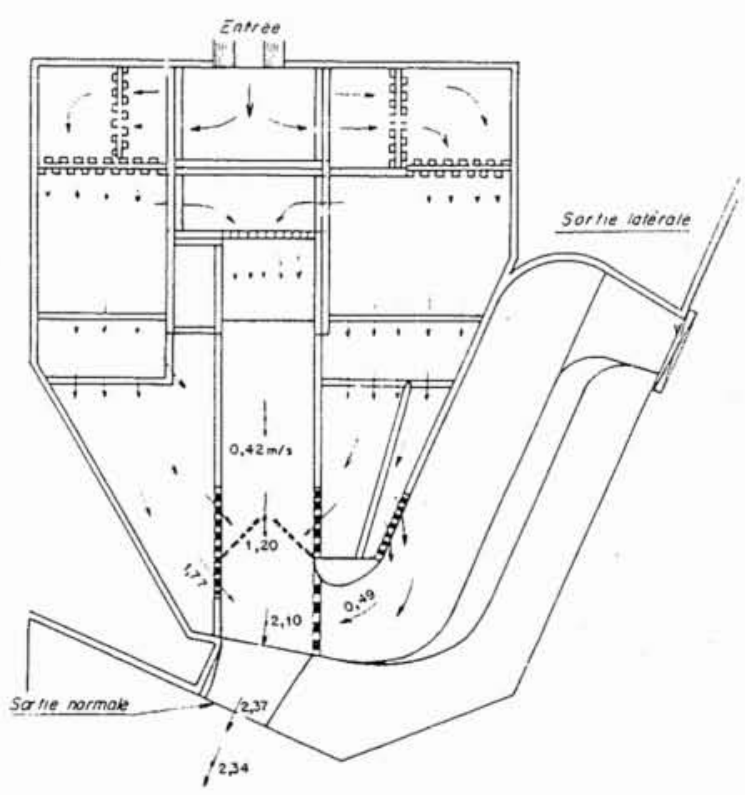

Figure 4 


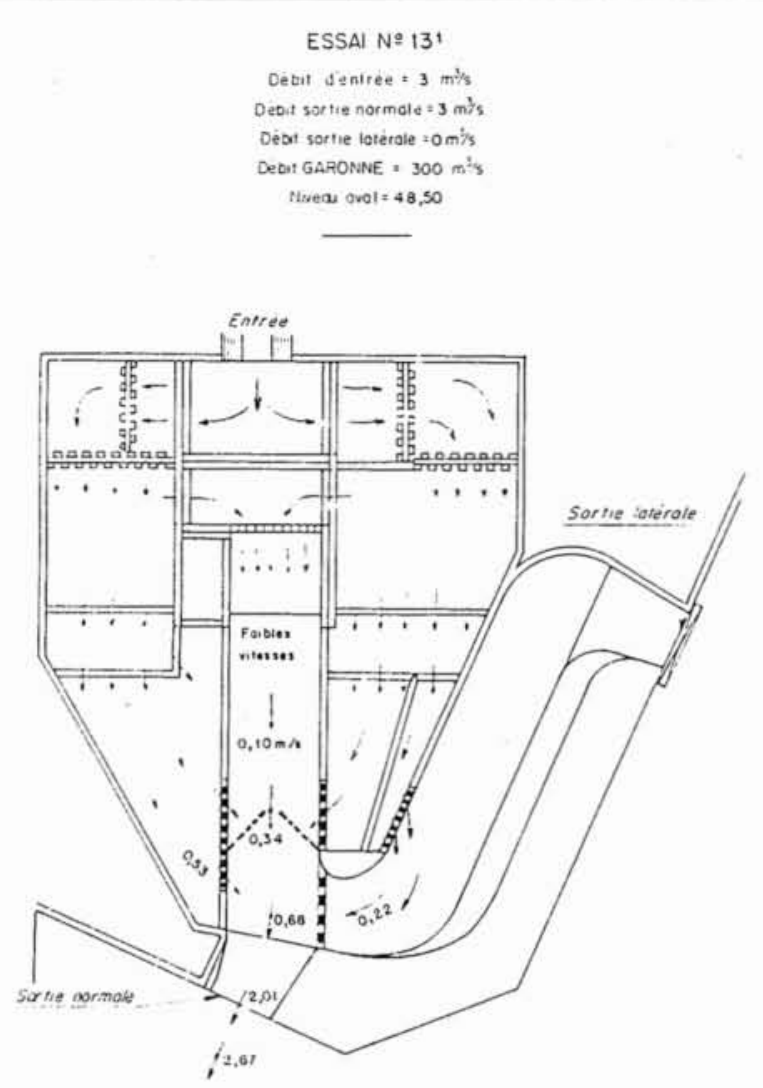

ESSAI N $13^{2}$

Debut dentree $=3$ mis

Debit sor the normale $=3 \mathrm{~m} / \mathrm{s}$

Debt sornie totérole $=0 \mathrm{~m} / \mathrm{s}$

DeBI GAFONAE $=300 \mathrm{~m}^{2} / \mathrm{s}$

Nriea aval $=40,50$
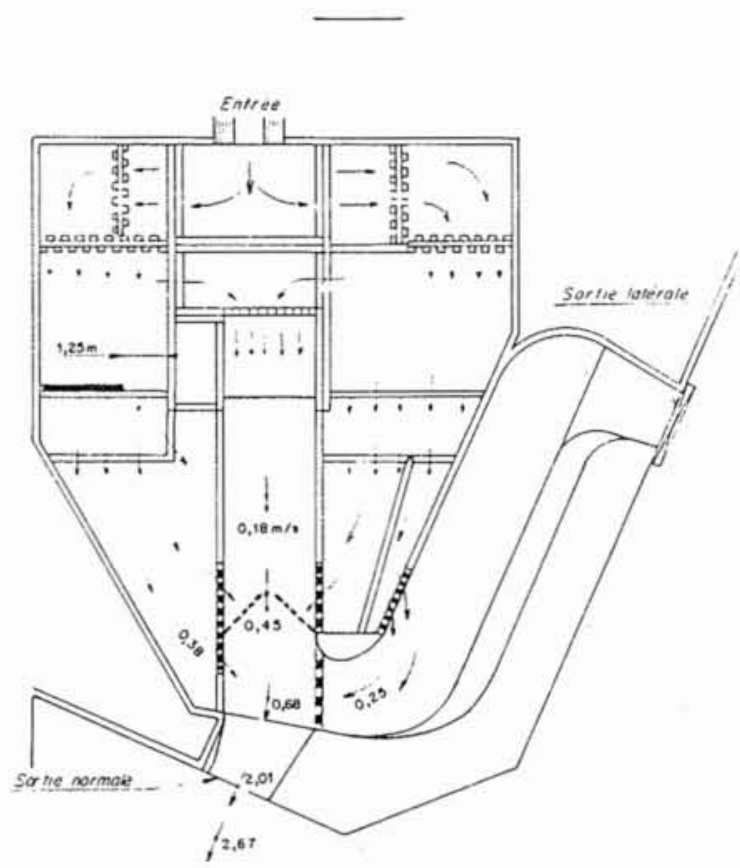

Figure 5

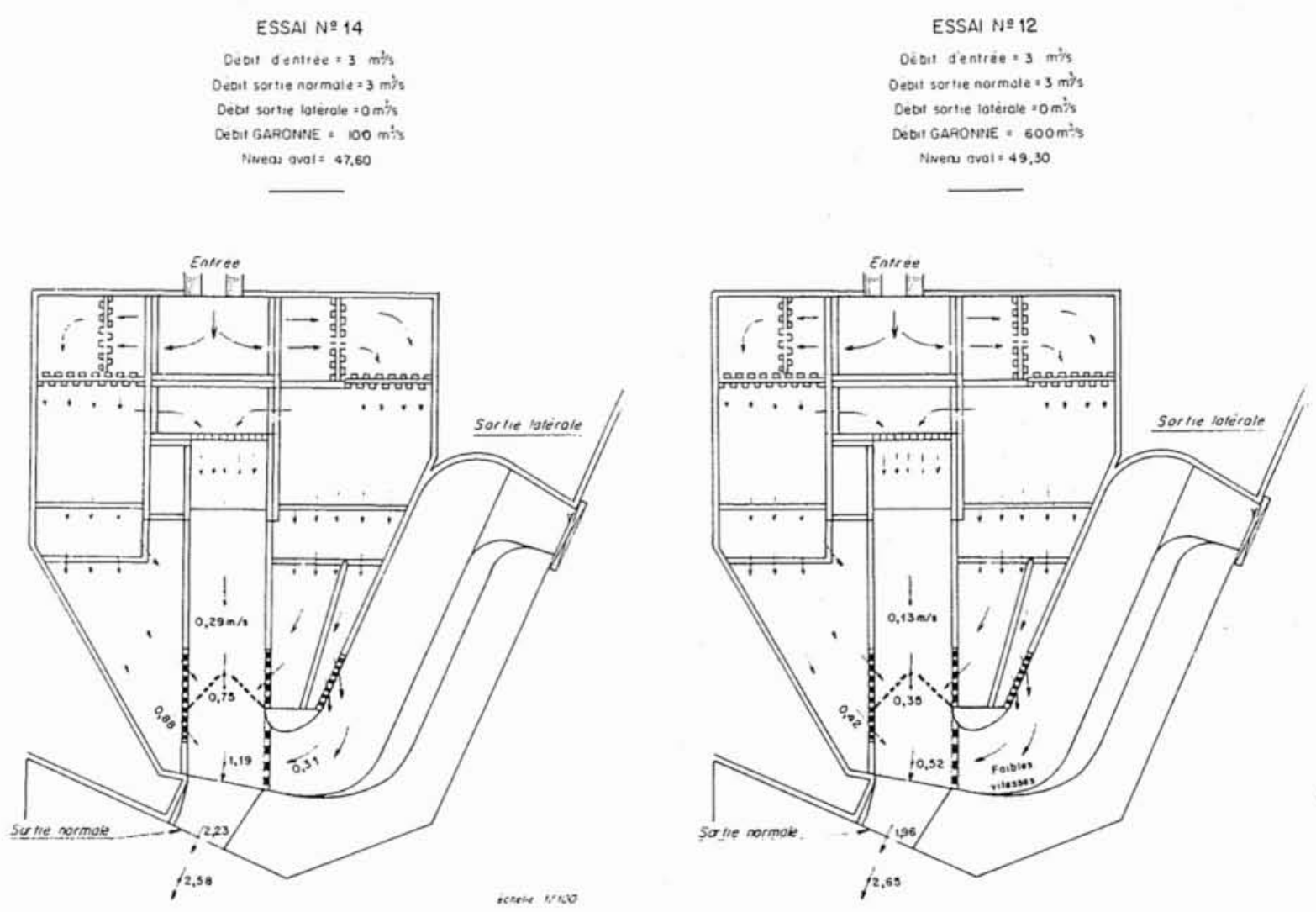

Figure 6 
ESSAI N 217

Dèbut dentrie 10 m's Debit sartie normale $=5 \mathrm{~m}$ 's Detat sortie laterole $/ 5 \mathrm{~m}^{2} \mathrm{~s}$ DeDit GARONNE $=300 \mathrm{~m}^{2} \mathrm{~s}$ Hivea aval $=48,50$

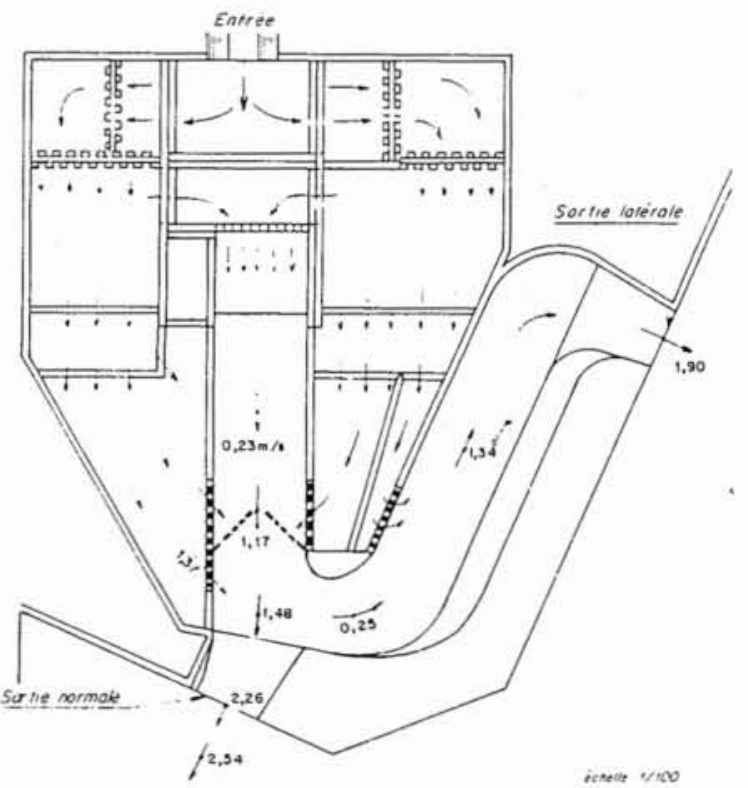

ESSAI NN 16

Debit deatree +10 mis

Deout sor tie normale is $\mathrm{m}$ is

Vebt sortie loterale $=5 \mathrm{mis}$

CeDi GAFOtIRE = $600 \mathrm{mi}$

titsen wats 49,30

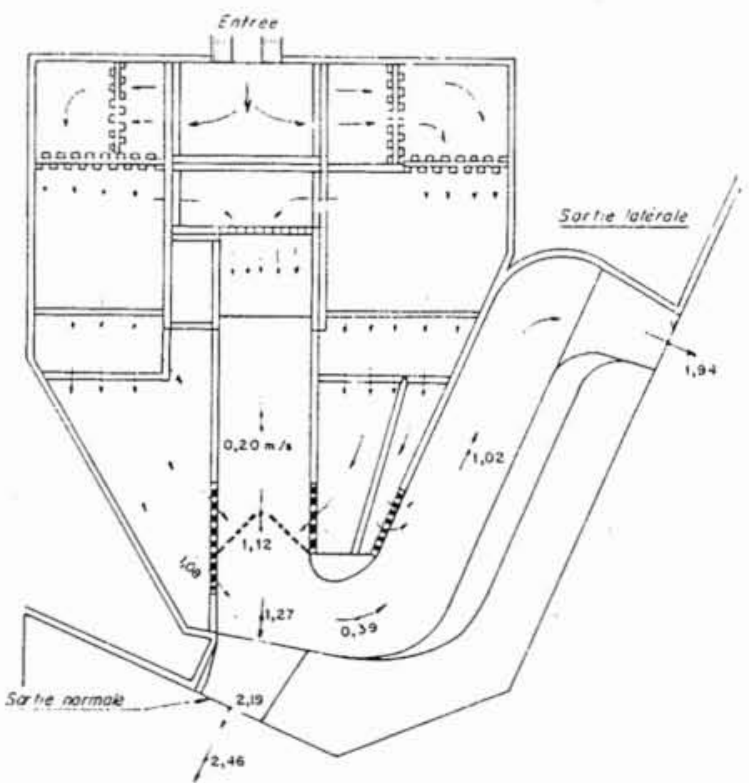

Figure 7

\section{Discussion}

Président : M. G. WEISROCK

M. TANE. - En ce qui concerne le suivi des aloses à GOLFECH, le problème sera différent quand la centrale fonctionnera.

Le Ministère de l'Environnement a donné rapidement l'autorisation demandée par E.D.F. pour exécuter les travaux en rivière nécessaires au rejet des eaux chaudes à la condition qu'un suivi biologique soit effectué. Quelles sont les dispositions prises pour ce suivi?

M. BELAUD. - Effectivement le mélange des eaux chaudes de la centrale avec les eaux de la rivière n'est pas instantané et peut influer sur le comportement des migrateurs.

Depuis 1980, des observations sont faites chaque année, actuellement par pêche, bientôt en utilisant la fenêtre d'observation de l'ascenseur. On pourra donc constater si la mise en service de la centrale en 1989 change quelque chose au comportement des migrateurs, et éventuellement prendre des mesures correctives.

M. TRIVELLATO. - Le choix de l'implantation d'un point de rejet des eaux chaudes a été fait sur une maquette réalisée à III.M.F.T. Les observations faites sur l'écoulement en maquette laissent penser que les rejets d'eau chaude de la centrale formeront des mèches d'eau chaude dans la rivière. L'homogénéisation des températures ne se faisant que plus loin en aval. Certains spécialistes pensent que cela peut être favorable au transit des migrateurs.

M. ROGUET. - Les observations faites à GOLFECH et BERGERAC montrent que la migration de l'alose se produit au-dessus de $16^{\circ}$ et est très sensible aux variations de température. On a constaté des pics journaliers de l'ordre de $10 \%$ de l'effectif total de migration. Il est important que le transit des aloses soit possible ces jours-là (par exemple, sur la Loire, le barrage de Blois doit être ouvert).

En ce qui concerne le transport des salmonidés, il existe une méthode qui utilise une boite spéciale isotherme sous eau, avec anesthesiant, et bulle d'oxygène ce qui évite le stress.

M. WEISROCK. - Ces études ont été menées lors des dispositions prises par E.D.F. pour rendre possible le franchissement de certains obstacles dans l'attente de la construction de passes à poissons. Ainsi durant les campagnes de migration, le laboratoire d'hychtiologie de l'Université de Toulouse a transporté les aloses en amont de GOLFECH, et le C.S.P. Toulouse a transporté les migrateurs en amont de Mauzac, à partir du piège de l'échelle de Bergerac.

M. CUINAT. - Certaines années de sécheresse, un transport a été fait sur l'Allier sur quelques centaines de kms. On a été déçu par le résultat car une partie des saumons redescendaient presque aussitôt. En plus du problème du stress, il semble qu'ils soient sensibles à un gradient d'odeur et n'aiment pas parcourir une trop grande distance en une seule fois.

Par conséquent le transport ne remplace pas les passes qui ont aussi leur utilité pour la dévalaison des juvéniles.

M. MORELLO. - Il est important, au moment des observations, de noter toutes les caractéristiques de l'environnement naturel (débits, températures, etc.) de façon à pouvoir les confronter aux théories actuelles sur le comportement des poissons.

M. BELAUD. - Les équipes relèvent soigneusement tous les paramètres accessibles. Cependant la température de l'eau reste le facteur primordial pour la mobilité du poisson. 